\title{
Does the distribution frequency matter? A subgroup specific analysis of the effectiveness of the EU School Fruit and Vegetable Scheme in Germany comparing twice and thrice weekly deliveries
}

\author{
Julia Haß ${ }^{1, *}$, Tanja Lischetzke ${ }^{2}$ and Monika Hartmann ${ }^{1}$ \\ ${ }^{1}$ Institute for Food and Resource Economics, Department of Agricultural and Food Market Research, University of \\ Bonn, Nussallee 21, 53115 Bonn, Germany: ${ }^{2}$ Department of Psychology, University of Koblenz-Landau, Landau, \\ Germany
}

Submitted 25 May 2017: Final revision received 26 0ctober 2017: Accepted 27 November 2017: First published online 26 January 2018

\begin{abstract}
Objective: The present study aimed to examine the effectiveness of two different implementation forms of the EU School Fruit and Vegetable Scheme (SFVS).

Design: A quasi-experimental design was applied including a thrice as well as a twice weekly intervention group. Repeated $24 \mathrm{~h}$ dietary recalls were used to measure children's fruit and vegetable $(F \& V)$ intake. Effects were analysed on days with and without F\&V deliveries using hierarchical linear regression models. Setting: Twelve primary schools in North Rhine-Westphalia, Germany.

Subjects: Third and fourth graders ( $n$ 664).

Results: Average daily F\&V intake at pre-intervention was 0.84 frequencies in the thrice weekly intervention group, 0.90 frequencies in the twice weekly intervention group and 1.25 frequencies in the control group. Providing children thrice weekly with $\mathrm{F} \& \mathrm{~V}$ increased children's F\&V intake on average by 0.96 $(P<0.001)$ frequencies/d. The effects were higher on days with $(1 \cdot 07 ; P<0.001)$ than on days without $(0.75 ; P<0.001) \mathrm{F} \& \mathrm{~V}$ deliveries. Distributing F\&V twice weekly resulted in an increase of $0.75(P<0.001)$ frequencies/d on average, again with higher effects on days with $(1.30 ; P<0.001)$ than without $(0.48 ; P<0.003)$ F\&V deliveries. Subgroup analysis revealed some indications for differential effectiveness only in the twice weekly intervention group.

Conclusions: The SFVS with thrice or twice weekly deliveries of F\&V led to a significant increase in children's F\&V intake on days with and without deliveries. The latter might provide an indication of positive long-term effects of the scheme. The scheme shows equal efficiency for almost all subgroups.
\end{abstract}

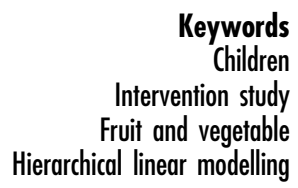

Children

Fruit and vegetable

Hierarchical linear modelling
Since childhood obesity has increased continuously in the past resulting in high prevalence rates in Europe and many other regions in the world ${ }^{(1,2)}$, changing dietary habits has become a public health goal. Even through the latest trends in childhood obesity show flattening in north-western Europe and first indications for stagnation in Germany, prevalence rates remain at a high level ${ }^{(3,4)}$. As eating habits established in childhood are likely to track into adulthood $^{(5,6)}$, improving dietary habits during early youth seems to be a promising prevention strategy ${ }^{(7-9)}$ and might reduce subsequent disease risk and health-care costs of obesity, which have become a serious concern ${ }^{(10,11)}$.

Empirical studies and literature reviews document that successful prevention strategies should be broad-based and well-integrated into children's lives ${ }^{(12,13)}$. Accordingly, the school setting is a natural fit for anti-obesity programmes and particularly effective when a multisector and bottom-up approach is applied ${ }^{(14-16)}$. Increasing the intake of fruits and vegetables (F\&V) has been shown to reduce long-term risk for obesity among adults, as incorporating them in the diet can reduce energy density, promote satiety and decrease energy intake ${ }^{(17-20)}$, and is therefore one key element of many prevention strategies. The objective of increasing F\&V intake also addresses the issue that children's average consumption level of these food items is still below the recommended intake ${ }^{(21,22)}$. The effectiveness of various school-based interventions programmes aimed at promoting $\mathrm{F} \& \mathrm{~V}$ intake has been previously summarized $^{(23,24)}$. It was concluded that most schemes were successful in increasing $F \& V$ intake of children. In these reviews, also intervention approaches providing children with free or subsidized F\&V were shown to be effective. These findings confirm those of previous empirical investigations in that accessibility and 
availability are considered to be strong predictors of children's F\&V consumption(25). Also more recent studies found that availability of F\&V at home mediated children's F\&V intake ${ }^{(26,27)}$. Additionally, research indicates that repeated exposure to a food is capable of increasing one's willingness to consume it ${ }^{(28)}$. Experimental studies with infants and children have provided strong evidence for the efficacy of exposure in increasing liking as well as intake of unfamiliar foods and drinks ${ }^{(29-32)}$. Availability as well as repeated exposure and thus increasing provision of healthy food choices in schools are therefore promising obesity prevention measures at the community level ${ }^{(13,33)}$

Against this background, the EU launched a School Fruit and Vegetables Scheme (SFVS) which provides children with free F\&V and thus encourages good eating habits in young people. The exact implementation of the scheme at national level is left to the member states but has to be based on national or regional strategies ${ }^{(34)}$. In North Rhine-Westphalia (NRW), the largest German federal state by population, the SFVS was launched towards the end of the 2009/10 academic year. Since its introduction, the number of schools participating in the scheme has increased continuously. The expansion was made possible due to an increase in the budget allocated to the programme and the reduction in the distribution frequency of F\&V deliveries from daily to thrice weekly ${ }^{(35,36)}$. In the school year 2012/13, when the present data were collected, the total budget for the SFVS NRW was 5 million Euro, with $50 \%$ being financed from federal state resources of NRW and $50 \%$ by EU funds ${ }^{(37)}$. The effectiveness of the SFVS NRW in terms of increasing children's F\&V intake was evaluated during the first year of implementation in 2010/11 by Methner et $a l .{ }^{(38)}$, confirming the success of daily $F \& V$ deliveries in increasing children's F\&V intake. However, since then there has been a reduction in $F \& V$ deliveries and this current implementation form with only thrice weekly deliveries has not been evaluated in the specific context of the SVFS NRW before. Thus, the question arises whether thrice weekly F\&V deliveries are sufficiently frequent to positively influence the dietary behaviour of children. Based on the assumption of a given budget, moreover, only a further reduction in F\&V distribution frequency, namely to twice weekly F\&V deliveries, will allow more schools' participation in the future. Thus, a closer look should also be taken at the effects of twice weekly F\&V deliveries. With thrice as well as twice weekly F\&V deliveries, it is expected that during one school week days without provision of F\&V will occur which have not been present before in NRW. This new situation raises the question whether effects that might be observed on days with F\&V deliveries are also verifiable on days without those deliveries. The latter would provide a first indication of the potential existence of long-term effects of the scheme, due to an increase in $\mathrm{F} \& \mathrm{~V}$ intake in the absence of F\&V deliveries. Finally, it seems important to investigate whether subgroups (e.g. boys $v$. girls, low $v$. high SES) are affected differently by both implementation forms, as it has been shown that F\&V intake is particularly low in boys and children with a low socioeconomic status $(\mathrm{SES})^{(21,22,39)}$.

Accordingly, the aim of the present study was to examine whether providing children F\&V thrice or twice weekly within the scope of the SFVS NRW is effective in increasing F\&V intake of participating children and relevant subgroups. Additionally, the study aims to give a first indication of possible long-term effects, by looking at the effects on days without $\mathrm{F} \& \mathrm{~V}$ deliveries.

\section{Methodology}

\section{Study design and data collection}

The study used a quasi-experimental design with a preintervention and post-intervention survey conducted among the same primary-school children (third and fourth grade, aged between 7 and 10 years at pre-intervention) before and after one year of participation in the SFVS NRW. Data were collected at the beginning and end of the 2012/13 school year. Overall, four schools with a thrice and four schools with a twice weekly distribution frequency of $\mathrm{F} \& \mathrm{~V}$, as well as four schools not participating in the SFVS NRW (control schools), were included in the evaluation study. The four schools with a thrice weekly distribution frequency were selected from schools which successfully applied for the admission in the programme in the 2012/13 academic year.* The four schools with a twice weekly distribution frequency were chosen out of the group of schools which applied for the admission in the programme, but were initially rejected. Criteria for inclusion were social and regional criteria as well as the concept of accompanying nutrition education measures to be established by the schools in the case of acceptance ${ }^{(37)}$. The four control schools were schools that had never applied for admission in the SFVS NRW. Selection criteria for all schools included in the study were their spatial proximity and accessibility for the research team as well as their size. The latter implied that schools considered for the study had to have at least two parallel classes in each grade to ensure a sufficient sample size. In schools with more than two classes in the third and fourth grade, respectively, classes were selected based on class teachers' willingness to participate in the survey. In total four classes of each school participated in the survey, resulting in forty-eight classes with 664 children included in the analysis. The study was carried out with the support of the Ministry for Climate Protection, Environment, Agriculture, Conservation and Consumer Protection of the State of North Rhine-Westphalia (MKULNV). $\dagger$ Furthermore,

\footnotetext{
* Selected by the MKULNV according to the EU framework ${ }^{(40)}$.

† The MKULNV provided access to the names of all schools that had applied for participation in the SFVS including those which were initially rejected.
} 
data handling was approved by the data protection officer of the University of Bonn.

\section{Survey instruments}

The F\&V intake of children was recorded in three repeated $24 \mathrm{~h}$ dietary recalls before as well as after one year of participation in the SFVS NRW, respectively. At both time points, the children completed a paper-and-pencil questionnaire as a class exercise, supervised by the research team at the first day of data collection. Using words and pictures, children were encouraged to recall the previous day and to describe the foods and drinks they consumed. For the analysis, all F\&V consumed over the course of the day were added up, using predefined coding strategies. At post-intervention, F\&V provided at school were included if children indicated that they have eaten $F \& V$ at school. Aspects of validity, reliability and sensitivity to change of this measurement were tested during the development of the questionnaire in the $\mathrm{UK}^{(41)}$. Furthermore, the version used in our survey has been adapted to the German school system and was successfully applied in the first evaluation study of the SFVS NRW carried out by Methner et $a l .{ }^{(38)}$. To ensure high data quality, manual data entry was done twice and inter-coder reliability was checked using Holsti's inter-coder reliability test which yielded an average inter-coder reliability score of 0.99 . Only questionnaires of children who filled in at least two $24 \mathrm{~h}$ dietary recalls at pre- and post-intervention were included in the analysis. Applying this strategy resulted in a sample size of 783 children. Reasonability of answers of these children was investigated carefully by outlier detection via boxplot analysis at school level. We defined outliers as values that were more than $1.5 \times$ interquartile range below the first quartile or more than $1.5 \times$ interquartile range above the third quartile. Following this definition fifty-three children were excluded from the analysis, resulting in a sample size of 730 children. We assessed SES by written questionnaires addressed to parents who filled in the survey at home on a voluntary basis. Children were assigned to low, middle or high SES applying the Brandenburger Social Index ${ }^{(42)}$. Written informed consent was obtained from all parents of included children. To be able to control for difference in SES, we had to exclude children with missing information on SES, due to missing information in the parent questionnaire, resulting in a final sample size of 664 children nested in forty-eight classes.

\section{Statistical analyses}

Differences between intervention groups with respect to demographic variables (proportion of children with low, middle and high SES, of third and fourth graders, of girls and boys, of children who eat lunch at home or at school) were analysed using $\chi^{2}$ tests. We used hierarchical linear regression models according to Raudenbush and Bryk ${ }^{(43)}$ to determine whether there were differences in F\&V intake change from the first measurement period (preintervention) to the post-intervention period (post-intervention) between control and intervention groups. We developed a four-step sequential modelling approach and fitted three-level models with days (level 1) nested in children (level 2), nested in classes (level 3). With only twelve schools in the sample we decided upon class as the highest level and treated the intervention variable as a class variable, given that the intervention was administered in classes. We refrained from adding dummy variables for schools because this would have resulted in a model with a very large number of additional fixed effects parameters (eleven main effects plus all interaction terms with the lower-level predictors). Bell et al. ${ }^{(44)}$ have shown for two-level models that a very small sample size (of 10 units) at the highest level can lead to a bias in fixed effects and, more importantly, is related to a very low power to detect non-zero fixed effects. However, to account for possible school differences in composition of children, we decided to include child- and class-level covariates, namely SES, gender and grade (explained in more detail below).

Model I was the 'empty' model without any explanatory variables besides a dummy variable (Period) for the postintervention period (post $=1$ ), which allows to partition the total variance in children's F\&V intake level into a variance between classes, children and time periods. The outcome $Y_{t i j}$ at level 1 refers to the F\&V intake (measured via $24 \mathrm{~h}$ recalls) on day $t$ of child $i$ in class $j$ :

Level 1 (days):

$$
Y_{t i j}=\pi_{0 i j}+\pi_{1 i j} \cdot \operatorname{Period}_{t i j}+e_{t i j}
$$

Level 2 (children):

$$
\begin{aligned}
& \pi_{0 i j}=\beta_{00 j}+r_{0 i j} \\
& \pi_{1 i j}=\beta_{10 j}+r_{1 i j}
\end{aligned}
$$

Level 3 (classes):

$$
\begin{aligned}
& \beta_{00 j}=\gamma_{000}+u_{00 j} \\
& \beta_{10 j}=\gamma_{100}+u_{10 j}
\end{aligned}
$$

Model II examined general intervention effects by adding a dummy-coded group membership variable for both intervention groups (control group =0). As the intervention was implemented on the class level, the intervention group variables were class variables and added as predictors in the level-3 equations. Accordingly, the level-1 and level-2 equations were the same as in Model I, while the level-3 equations were:

Level 3 (classes):

$$
\begin{aligned}
& \beta_{00 j}=\gamma_{000}+\gamma_{001} \cdot \text { Int.thrice }_{j}+\gamma_{002} \cdot \text { Int.twice }_{j}+u_{00 j} \\
& \beta_{10 j}=\gamma_{100}+\gamma_{101} \cdot \text { Int.thrice }_{j}+\gamma_{102} \cdot \text { Int.twice } j+u_{10 j}
\end{aligned}
$$


For an increase in $\mathrm{F} \& \mathrm{~V}$ intake, due to the intervention, we expect $\gamma_{101}$ and $\gamma_{102}$ - that is, the mean differences between the groups (intervention group minus control group) with respect to the differences between the periods (intervention period minus control period) - to be significantly larger than zero ${ }^{(45)}$.

Model III additionally included level-2 covariates to control for compositional effects on the class level (e.g. clustering of children of high SES within a certain class). On the child level (level 2), a dummy variable capturing whether the children usually eat lunch at school $(=0)$ or at home $(=1)$, gender (boys $=0$, girls $=1$ ), and a dummy variable for low SES and middle SES, respectively, with high SES as the reference group $(=0)$, were considered as covariates and centred around the grand mean. Centring a level-2 (child-level) dummy variable around the grand mean yields level-3 (class) coefficients that can be interpreted as adjusted outcome means ${ }^{(46)}$. Additionally, grade (third grade $=-0 \cdot 5$, fourth grade $=+0 \cdot 5$ ) was included at the class level (level 3) as an effect-coded covariate in each of the two level- 3 equations, so that it can be tested whether group differences in $F \& V$ intake change exist beyond grade effects. The model equations were:

Level 1 (days):

$$
Y_{t i j}=\pi_{0 i j}+\pi_{1 i j} \cdot \text { Period }_{t i j}+e_{t i j}
$$

Level 2 (children):

$$
\begin{aligned}
\pi_{0 i j}= & \beta_{00 j}+\beta_{01 j} \cdot \text { Lunch }_{i j}+\beta_{02 j} \cdot \text { Gender }_{i j} \\
& +\beta_{03 j} \cdot \text { SES. }_{\text {low }}+\beta_{04 j} \cdot \text { SES. middle }_{i j}+r_{0 i j}(9) \\
\pi_{1 i j}= & \beta_{10 j}+\beta_{11 j} \cdot \text { Lunch }_{i j}+\beta_{12 j} \cdot \text { Gender }_{i j} \\
& +\beta_{13 j} \cdot \text { SES. } \text { low }_{i j}+\beta_{14 j} \cdot \text { SES. } \text { middle }_{i j}+r_{1 i j}(10)
\end{aligned}
$$

Level 3 (classes):

$$
\begin{gathered}
\beta_{00 j}=\gamma_{000}+\gamma_{001} \cdot \text { Int.thrice }_{j}+\gamma_{002} \cdot \text { Int.twice }_{j} \\
+\gamma_{003} \cdot \text { Grade }_{j}+u_{00 j} \\
\beta_{01 j}=\gamma_{010}+u_{01 j} \\
\beta_{02 j}=\gamma_{020}+u_{02 j} \\
\beta_{03 j}=\gamma_{030}+u_{03 j} \\
\beta_{04 j}=\gamma_{040}+u_{04 j} \\
\beta_{10 j}=\gamma_{100}+\gamma_{101} \cdot \text { Int }_{\text {thrice }}+\gamma_{102} \cdot \text { Int twice }_{j} \\
+\gamma_{103} \cdot \text { Grade }_{j}+u_{10 j} \\
\beta_{11 j}=\gamma_{110}+u_{11 j} \\
\beta_{12 j}=\gamma_{120}+u_{12 j} \\
\beta_{13 j}=\gamma_{130}+u_{13 j} \\
\beta_{14 j}=\gamma_{140}+u_{14 j}
\end{gathered}
$$

For each of the covariates, the deviance statistic was used to test which parameter should be included as a random effect ${ }^{(47)}$. Only significant random slopes were retained in the final model.

In Model III, the level-3 coefficients that capture the intervention effects on the change in F\&V intake from preto post-intervention (i.e. $\gamma_{101}$ and $\gamma_{102}$ ) can be interpreted in the following way: $\gamma_{101}\left(\gamma_{102}\right)$ represents the mean difference between the thrice (twice) weekly intervention group and the control group with respect to the difference between the intervention and the control period, adjusted for differences in the proportion of compared groups (i.e. girls, children with low/middle SES, children who eat lunch at home) across classes. Grade was effect-coded so that the meaning of the estimated scores for the control group (i.e. $\gamma_{000}$, the pre-intervention scores, and $\gamma_{100}$, the pre - post difference scores) does not change from Model II to Model III.

Additionally, we explored whether subgroups were affected differently by the school $\mathrm{F} \& \mathrm{~V}$ intervention (i.e. differential effectiveness of the intervention). To do that, we examined the cross-level interactions by adding the intervention group variables to each of the concerned level-3 equations (e.g. to examine whether children usually eating in the school canteen were affected differently by the school F\&V intervention, group membership variables were added to equations (12) and (17)). This analysis was done in a stepwise procedure. First, the crosslevel interaction with each covariate was tested one after another. Second, significant cross-level interactions were combined in one model to scrutinize the unique contribution of each interaction term. Due to the explorative character of this analysis, we accepted a significance level of $10 \%$ for this part of our analysis.

Finally, we tested whether the two intervention groups (twice $v$. thrice weekly deliveries) differed with respect to the increase in $\mathrm{F} \& \mathrm{~V}$ intake from pre- to post-intervention by analysing the data of the two intervention groups only (i.e. Model II was adapted so that only one dummy variable for twice $v$. thrice weekly intervention was included at level 3).

All analyses described above were carried out (i) jointly for days with and without F\&V deliveries, (ii) for days with and (iii) for days without F\&V deliveries. Children in schools with a thrice weekly distribution frequency filled in the $24 \mathrm{~h}$ dietary recall for two days with and one day without F\&V deliveries, whereas children in schools with the twice weekly distribution frequency filled in the $24 \mathrm{~h}$ recall for one day with and two days without F\&V deliveries, respectively. This allowed us to evaluate both the overall effect in the two intervention groups considering the differences in distribution frequency of school F\&V, as well as differences in effects between days with and without F\&V deliveries. Consequently, at pre-intervention for the control and both intervention groups and at postintervention for the control group, the analysis was always based on three $24 \mathrm{~h}$ dietary recalls. This also holds in the case of the joint analysis of days with and without F\&V 
deliveries for both intervention groups at postintervention. However, for the analysis of days with $\mathrm{F} \& \mathrm{~V}$ deliveries we considered at post-intervention only the respective days with $\mathrm{F} \& \mathrm{~V}$ deliveries (two $24 \mathrm{~h}$ dietary recalls in the case of schools with thrice weekly deliveries, one $24 \mathrm{~h}$ dietary recalls in the case of schools with thrice weekly deliveries). Analogously, for studying the impact of the SFVS on days without F\&V deliveries we considered at post-intervention only the respective days without $\mathrm{F} \& \mathrm{~V}$ deliveries (one $24 \mathrm{~h}$ dietary recall in the case of schools with thrice weekly deliveries, two $24 \mathrm{~h}$ dietary recalls in the case of schools with thrice weekly deliveries).

\section{Results}

\section{Characteristics of children}

The final sample consisted of 664 primary-school children, who filled in two or more $24 \mathrm{~h}$ dietary recalls for the preand post-intervention measurement, respectively, without missing values for the considered control variables. Demographic characteristics of the three groups (children in twice/thrice weekly intervention groups and control group) are summarized in Table 1. Groups did not show significant differences regarding grade and gender, but did so with respect to SES and the proportion of children usually eating lunch at home. The proportion of children with high SES was higher in the control group than in the intervention groups. Furthermore, children with low SES were over-represented in the intervention group with the thrice weekly school F\&V deliveries. The proportion of children eating lunch at home was lowest in the control group and highest in the thrice weekly intervention group. The identified differences between the groups clearly show the need for controlling for potential confounders in the following analysis.

\section{Overall intervention effect of school fruit and vegetable deliveries}

Results of the series of hierarchical linear regression models analysing the overall intervention effect on daily F\&V intake of children are presented in Table 2. The empty model (Model I) showed that, on average, children's daily F\&V intake frequency increased by 0.59 $\left(\gamma_{100}\right)$ from $1.00\left(\gamma_{000}\right)$ at pre-intervention to 1.59 at postintervention. To explain this increase by the school F\&V intervention, dummy-coded group membership variables were added to the two level-3 (classes) equations. Intervention groups differed significantly in the mean preintervention scores, with an average daily $\mathrm{F} \& \mathrm{~V}$ intake frequency of 1.25 in the control group $\left(\gamma_{000}\right), 0.84$ $\left(\gamma_{000}+\gamma_{001}\right)$ in the thrice weekly intervention group and $0 \cdot 90\left(\gamma_{000}+\gamma_{002}\right)$ in the twice weekly intervention group. That is, the control group had a higher average F\&V intake before the intervention. With respect to the change in daily F\&V intake from pre- to post-intervention, the intervention groups showed higher increases than the control group: children in the control group did not significantly increase their daily $\mathrm{F} \& \mathrm{~V}$ intake frequency $\left(\gamma_{100}=0.03, P=0.789\right)$. The thrice weekly intervention group's increase was significantly larger than the control group's increase (by $\left.\gamma_{101}=0.96\right)$. That is, the thrice weekly intervention group increased their F\&V intake by $0.99\left(\gamma_{100}+\gamma_{101}\right)$, from 0.84 frequencies/d at pre-intervention to 1.83 frequencies/d at post-intervention. In a similar vein, the twice weekly intervention group's increase was significantly larger than the control group's increase (by $\gamma_{102}=0.75$ ). That is, the twice weekly intervention group increased their $\mathrm{F} \& \mathrm{~V}$ intake by 0.78 frequencies/d $\left(\gamma_{100}+\gamma_{102}\right)$, from 0.90 frequencies/d at pre-intervention to 1.68 frequencies/d at post-intervention. Adding the group membership dummy variables to the level-3 equations in Model II reduced the variance of the level-3 residuals of slopes for period $\left(u_{10 j}\right)$.

Table 1 Demographic characteristics of the study sample at pre-intervention: third and fourth grade children $(n$ 664) from twelve primary schools in North Rhine-Westphalia, Germany, beginning of the 2012/13 school year

\begin{tabular}{|c|c|c|c|c|c|c|c|c|c|}
\hline & \multicolumn{2}{|c|}{$\begin{array}{l}\text { Thrice weekly } \\
\text { (total } N 183 \text { ) }\end{array}$} & \multicolumn{2}{|c|}{$\begin{array}{l}\text { Twice weekly } \\
\text { (total N 250) }\end{array}$} & \multicolumn{2}{|c|}{$\begin{array}{c}\text { Control } \\
\text { (total } N 231 \text { ) }\end{array}$} & \multirow[b]{2}{*}{$x^{2}$} & \multirow[b]{2}{*}{$P$ value } & \multirow[b]{2}{*}{$\mathrm{df}$} \\
\hline & $n$ & $\%$ & $n$ & $\%$ & $n$ & $\%$ & & & \\
\hline \multicolumn{10}{|l|}{ Grade } \\
\hline 3rd grade & 83 & $45 \cdot 4$ & 127 & $50 \cdot 8$ & 128 & 55.4 & & & \\
\hline 4th grade & 100 & $54 \cdot 6$ & 123 & $49 \cdot 2$ & 103 & 44.6 & $4 \cdot 13$ & 0.127 & 2 \\
\hline \multicolumn{10}{|l|}{ Gender } \\
\hline Girl & 100 & $54 \cdot 6$ & 124 & $49 \cdot 6$ & 101 & 43.7 & & & \\
\hline Boy & 83 & $45 \cdot 4$ & 126 & 50.4 & 130 & $56 \cdot 3$ & 4.94 & 0.084 & 2 \\
\hline \multicolumn{10}{|c|}{ Lunch at home } \\
\hline Yes & 133 & $72 \cdot 7$ & 136 & 54.4 & 110 & 47.6 & & & \\
\hline No & 50 & $27 \cdot 3$ & 114 & $45 \cdot 6$ & 121 & 52.4 & 27.35 & 0.000 & 2 \\
\hline \multicolumn{10}{|l|}{ SES* } \\
\hline Low & 74 & $40 \cdot 4$ & 39 & $15 \cdot 6$ & 19 & 8.2 & & & \\
\hline Middle & 52 & $28 \cdot 4$ & 63 & $25 \cdot 2$ & 55 & 23.8 & 84.86 & 0.000 & 4 \\
\hline High & 57 & $31 \cdot 1$ & 148 & $59 \cdot 2$ & 157 & 68.0 & & & \\
\hline
\end{tabular}

SES, socio-economic status.

${ }^{*}$ Brandenburger Social Index of Böhm et al. ${ }^{(42)}$. 
Table 2 Overall intervention effect of fruit and vegetable (F\&V) deliveries of the European School Fruit Scheme in North Rhine-Westphalia, Germany, on the F\&V intake of third and fourth grade children ( $n 664)$ from twelve primary schools, beginning to the end of the $2012 / 13$ school year

\begin{tabular}{|c|c|c|c|c|c|c|c|c|c|}
\hline & \multicolumn{6}{|c|}{ Fixed part } & \multicolumn{3}{|c|}{ Random part } \\
\hline & Coef. & Est. & SE & $t$ & $P$ value & df & Coef. & Var. & $P$ value \\
\hline \multicolumn{10}{|l|}{ Model I } \\
\hline Intercept & $\gamma_{000}$ & 1.00 & 0.05 & $16 \cdot 80$ & $<0.001$ & 47 & $u_{00 j}$ & 0.13 & $<0.001$ \\
\hline For period slope & & & & & & & & & \\
\hline Intercept & $\gamma_{100}$ & 0.59 & 0.09 & $6 \cdot 88$ & $<0.001$ & 47 & $u_{10 j}$ & 0.28 & $<0.001$ \\
\hline \multicolumn{10}{|l|}{ Model II } \\
\hline Intercept & $Y_{000}$ & $1 \cdot 25$ & 0.09 & $13 \cdot 62$ & $<0.001$ & 45 & $u_{00 j}$ & 0.09 & $<0.001$ \\
\hline Thrice weekly intervention & $Y_{001}$ & -0.41 & 0.13 & -3.05 & 0.004 & 45 & & & \\
\hline Twice weekly intervention & $Y_{002}$ & -0.35 & 0.13 & -2.69 & 0.01 & 45 & & & \\
\hline \multicolumn{10}{|l|}{ For period slope } \\
\hline Intercept & $\gamma_{100}$ & 0.03 & $0 \cdot 10$ & 0.30 & 0.767 & 45 & $u_{10 j}$ & 0.11 & $<0.001$ \\
\hline Thrice weekly intervention & $\gamma_{101}$ & 0.96 & $0 \cdot 15$ & $6 \cdot 34$ & $<0.001$ & 45 & & & \\
\hline \multirow{2}{*}{\multicolumn{10}{|c|}{ Model III }} \\
\hline & & & & & & & & & \\
\hline Intercept & $\gamma_{000}$ & $1 \cdot 20$ & 0.08 & 14.75 & $<0.001$ & 44 & $u_{00 j}$ & 0.08 & $<0.001$ \\
\hline Thrice weekly intervention & $Y_{001}$ & -0.29 & 0.12 & $-2 \cdot 40$ & 0.021 & 44 & & & \\
\hline Twice weekly intervention & $\gamma_{002}$ & -0.31 & $0 \cdot 11$ & $-2 \cdot 83$ & 0.007 & 44 & & & \\
\hline Grade & $\gamma_{003}$ & -0.38 & 0.09 & $-4 \cdot 12$ & $<0.001$ & 44 & & & \\
\hline Lunch at home & $Y_{010}$ & -0.31 & 0.06 & $-5 \cdot 26$ & $<0.001$ & 513 & & & \\
\hline Gender & $Y_{020}$ & 0.32 & 0.06 & $5 \cdot 58$ & $<0.001$ & 513 & & & \\
\hline Low SES & $\gamma_{030}$ & $-0 \cdot 18$ & 0.08 & $-2 \cdot 18$ & 0.03 & 513 & & & \\
\hline Middle SES & $\gamma_{040}$ & $-0 \cdot 19$ & 0.07 & $-2 \cdot 75$ & 0.006 & 513 & & & \\
\hline \multicolumn{10}{|l|}{ For period slope } \\
\hline Intercept & $\gamma_{100}$ & 0.03 & $0 \cdot 10$ & 0.27 & 0.789 & 44 & $u_{10 j}$ & $0 \cdot 10$ & $<0.001$ \\
\hline Thrice weekly intervention & $\gamma_{101}$ & 0.99 & $0 \cdot 15$ & $6 \cdot 70$ & $<0.001$ & 44 & & & \\
\hline Twice weekly intervention & $\gamma_{102}$ & 0.77 & 0.14 & $5 \cdot 71$ & $<0.001$ & 44 & & & \\
\hline Grade & $\gamma_{103}$ & 0.17 & $0 \cdot 11$ & 1.47 & 0.148 & 513 & & & \\
\hline Lunch at home & $\gamma_{110}$ & 0.12 & 0.08 & 1.46 & 0.145 & 513 & & & \\
\hline Gender & $\gamma_{120}$ & 0.16 & 0.09 & 1.76 & 0.085 & 47 & $u_{12 j}$ & $0 \cdot 10$ & 0.021 \\
\hline Low SES & $\gamma_{130}$ & $-0 \cdot 10$ & 0.11 & -0.92 & 0.358 & 513 & & & \\
\hline Middle SES & $\gamma_{140}$ & 0.01 & 0.09 & $0 \cdot 11$ & 0.913 & 513 & & & \\
\hline
\end{tabular}

Coef., coefficient; Est., estimate, Var., variance components; SES, socio-economic status.

The variance of the level-1 residual $\left(e_{t i j}\right)$ was estimated to be 1.08 in all models. The variance of the level-2 residual of intercepts $\left(r_{0 i j}\right)$ was 0.20 in Models I and II and 0.14 in Model III. The variance of the level-2 residual of slopes for period $\left(r_{1 i j}\right)$ was 0.20 in Models I and II and 0.17 in Model III.

As a measure of effect size for the effect of group membership on change in F\&V intake, we calculated the proportional reduction in level-3 variance of slopes for period when the two group dummy variables were added to the model ${ }^{(47)}$, resulting in a quasi- $R^{2}$ of $61 \cdot 80 \%$, which can be considered a large effect.

In Model III, we added the grand-mean-centred childlevel covariates (SES, gender, lunch at home) on level 2 (children) as well as effect-coded grade on level 3 (classes). The results can be found in the lower part of Table 2. By adding the child-level covariates grand-meancentred, differences between classes with respect to compositional effects (i.e. proportion of girls, children with low/ middle SES, children who eat lunch at home) are adjusted for. The results of Model III showed that the overall effectiveness of the school F\&V intervention was confirmed when controlling for compositional effects and for grade. In this model, slopes for gender were treated as random $\left(u_{12 j}\right)$. The adjusted F\&V intake scores at pre-intervention and the adjusted F\&V intake change scores (i.e. the expected scores with respect to the proportion of girls, of children with low/middle SES and of children who eat lunch at home being identical across classes) can be calculated from the fixed effects in Modell III, as explained above. The 'main effects' of the control variables in this model (gender, SES, lunch at home, grade) represent mean differences with respect to the intercept (i.e. effects on pre-intervention scores). The results revealed that children who usually eat lunch at home, children from homes with low and middle SES as well as in fourth grade had a significantly lower intake score at pre-intervention compared with their reference group. Girls showed significantly higher F\&V intake scores at pre-intervention than boys.

Moreover, in a separate model that extends Model III, we explored whether the school F\&V intervention affected subgroups of children differently. To do so, we examined the cross-level interactions between the group membership variables on level 3 and each child-level covariate on level 2. This analysis revealed that the twice weekly intervention group's increase in F\&V intake was 0.31 frequencies/d lower for children who usually eat lunch at home compared with children who eat lunch at school $(P=0.085$; see online supplementary material, Supplemental Table 1). For the thrice weekly intervention group, the coefficient was also negative $(-0 \cdot 22)$ but non-significant $(P=0 \cdot 297)$. No other cross-level interaction reached significance. 


\section{Intervention effect on days with school fruit and vegetable deliveries}

Besides the overall intervention effect, we were also interested in the effectiveness of the SFVS in increasing children's F\&V intake on days with and without school F\&V deliveries. The effect on days with F\&V deliveries can be interpreted as a direct effect due to the increase in F\&V availability in schools (structural prevention), whereas an increase in $F \& V$ intake on days without school F\&V deliveries might be considered a first indication of possible long-term effects of the SFVS. Table 3 presents the results of the series of hierarchical linear regression models in which only the days with $\mathrm{F} \& \mathrm{~V}$ deliveries were included for the intervention groups in the post-intervention period. The empty model (Model I) showed that, on average, children's F\&V intake frequency increased by $0.79\left(\gamma_{100}\right)$ from $1.00\left(\gamma_{000}\right)$ at pre-intervention to 1.79 at postintervention on days with school F\&V deliveries. As can be seen in Table 3 (Model II), on school F\&V days the thrice weekly intervention group's increase was significantly larger than the control group's increase (by $\left.\gamma_{101}=1.07\right)$. That is, the thrice weekly intervention group increased their $\mathrm{F} \& \mathrm{~V}$ intake by $1 \cdot 10 \quad\left(\gamma_{100}+\gamma_{101}\right)$ frequencies/d, from 0.84 frequencies/d at pre-intervention to 1.94 frequencies/d at post-intervention. In a similar vein, the twice weekly intervention group's increase was significantly larger than the control group's intake (by $\gamma_{102}=1 \cdot 30$ ). That is, the twice weekly intervention group increased their $\mathrm{F} \& \mathrm{~V}$ intake by $1.33\left(\gamma_{100}+\gamma_{102}\right)$ frequencies/d, from 0.90 frequencies/d at pre-intervention to 2.23 frequencies/d at post-intervention. The effect size for the effect of group membership on change in $F \& V$ intake (i.e. quasi- $R^{2}$ for the reduction in level-3 variance of slopes for period) was $70.58 \%$. Adjusting for differences between classes with respect to level-2 (children) compositional effects (gender, SES, lunch at home) and controlling for grade (on level 3) in Model III confirmed the overall effectiveness of the school F\&V intervention on school F\&V days (see Table 3). Comparing Tables 2 and 3 reveals that the intervention effect in the twice as well as the thrice weekly intervention group was considerably higher on days with school F\&V deliveries compared with the effect considering days with and without F\&V deliveries.

Again, as an extension of Model III, we explored whether subgroups of children were affected differently by the school F\&V intervention focusing this time on school F\&V

Table 3 Intervention effect on days with fruit and vegetable (F\&V) deliveries of the European School Fruit Scheme in North Rhine-Westphalia, Germany, on the F\&V intake of third and fourth grade children $(n 664)$ from twelve primary schools, beginning to the end of the 2012/13 school year

\begin{tabular}{|c|c|c|c|c|c|c|c|c|c|}
\hline & \multicolumn{6}{|c|}{ Fixed part } & \multicolumn{3}{|c|}{ Random part } \\
\hline & Coef. & Est. & SE & $t$ & $P$ value & df & Coef. & Var. & $P$ value \\
\hline \multicolumn{10}{|l|}{ Model I } \\
\hline Intercept & $\gamma_{000}$ & 1.00 & 0.06 & $16 \cdot 84$ & $<0.001$ & 47 & $u_{00 j}$ & 0.13 & $<0.001$ \\
\hline For period slope & & & & & & & & & \\
\hline Intercept & $y_{000}$ & 1.25 & 0.09 & $13 \cdot 63$ & $<0.001$ & 45 & $u_{0 n i}$ & 0.09 & $<0.001$ \\
\hline Thrice weekly intervention & $\gamma_{001}$ & -0.41 & 0.13 & -3.06 & 0.004 & 45 & $u_{00 j}$ & 0.05 & $<0.001$ \\
\hline Twice weekly intervention & $\gamma_{002}$ & -0.35 & 0.13 & -2.70 & 0.01 & 45 & & & \\
\hline \multicolumn{10}{|l|}{ For period slope } \\
\hline Intercept & $\gamma_{100}$ & 0.03 & 0.11 & 0.26 & 0.799 & 45 & $u_{10 j}$ & 0.14 & $<0.001$ \\
\hline Thrice weekly intervention & $\gamma_{101}$ & 1.07 & 0.17 & $6 \cdot 27$ & $<0.001$ & 45 & & & \\
\hline Twice weekly intervention & $\gamma_{102}$ & 1.30 & 0.17 & 7.68 & $<0.001$ & 45 & & & \\
\hline \multicolumn{10}{|l|}{ Model III } \\
\hline Intercept & $\gamma_{000}$ & 1.21 & 0.08 & 14.65 & $<0.001$ & 44 & $u_{00 j}$ & 0.08 & $<0.001$ \\
\hline Thrice weekly intervention & $\gamma_{001}$ & -0.30 & 0.12 & -2.43 & 0.019 & 44 & & & \\
\hline Twice weekly intervention & $\gamma_{002}$ & -0.32 & 0.11 & -2.82 & 0.007 & 44 & & & \\
\hline Grade & $\gamma_{003}$ & -0.37 & 0.10 & -3.92 & $<0.001$ & 44 & & & \\
\hline Lunch at home & $\gamma_{010}$ & -0.31 & 0.06 & $-5 \cdot 26$ & $<0.001$ & 513 & & & \\
\hline Gender & $\gamma_{020}$ & 0.31 & 0.06 & 5.57 & $<0.001$ & 513 & & & \\
\hline Low SES & $\gamma_{030}$ & -0.18 & 0.08 & $-2 \cdot 18$ & 0.03 & 513 & & & \\
\hline Middle SES & $\gamma_{040}$ & -0.19 & 0.07 & -2.75 & 0.006 & 513 & & & \\
\hline \multicolumn{10}{|l|}{ For period slope } \\
\hline Intercept & $\gamma_{100}$ & 0.03 & 0.11 & 0.25 & 0.805 & 44 & $u_{10 j}$ & 0.13 & $<0.001$ \\
\hline Thrice weekly intervention & $\gamma_{101}$ & 1.09 & 0.17 & $6 \cdot 32$ & $<0.001$ & 44 & & & \\
\hline Twice weekly intervention & $\gamma_{102}$ & 1.31 & 0.17 & 7.95 & $<0.001$ & 44 & & & \\
\hline Grade & $\gamma_{103}$ & 0.19 & 0.14 & 1.36 & 0.181 & 44 & & & \\
\hline Lunch at home & $\gamma_{110}$ & 0.11 & 0.09 & $1 \cdot 16$ & 0.248 & 513 & & & \\
\hline Gender & $\gamma_{120}$ & 0.15 & 0.11 & 1.37 & 0.177 & 47 & $u_{12 j}$ & 0.21 & $<0.001$ \\
\hline Low SES & $\gamma_{130}$ & -0.14 & 0.12 & $-1 \cdot 11$ & 0.268 & 513 & & & \\
\hline Middle SES & $\gamma_{140}$ & -0.05 & 0.10 & -0.45 & 0.652 & 513 & & & \\
\hline
\end{tabular}

Coef., coefficient; Est., estimate; Var., variance components; SES, socio-economic status.

The variance of the level-1 residual $\left(e_{t i j}\right)$ was estimated to be 0.94 in all models. The variance of the level- 2 residual of intercepts $\left(r_{0 i j}\right)$ was 0.24 in Models I and II and 0.19 in Model III. The variance of the level-2 residual of slopes for period $\left(r_{1 i j}\right)$ was 0.36 in Models I and II and 0.30 in Model III. 
days only. This analysis revealed that on days with school $\mathrm{F} \& \mathrm{~V}$ deliveries, the twice weekly intervention group's increase in $\mathrm{F} \& \mathrm{~V}$ intake was 0.40 frequencies/d lower for children who usually eat lunch at home than for children who eat lunch at school $(P=0.062)$ and 0.44 frequencies/d lower for children with middle SES $(P=0.079)$ compared with children with high SES (see online supplementary material, Supplemental Table 2). For the thrice weekly intervention group, the corresponding coefficients were also negative but non-significant (lunch at home: $-0 \cdot 15$, $P=0.519$; middle SES: $-0 \cdot 14, P=0.595)$.

\section{Intervention effect on days without school fruit and vegetable deliveries}

Table 4 presents the results of the series of hierarchical linear regression models in which only the days without $\mathrm{F} \& \mathrm{~V}$ deliveries were included for the post-intervention period. The empty model (Model I) showed that on average children's F\&V intake frequency on days without school F\&V deliveries increased by $0.41\left(\gamma_{100}\right)$ from 1.00 $\left(\gamma_{000}\right)$ at pre-intervention to 1.41 at post-intervention, and thus considerably less than if all days are considered ( $0 \cdot 59$; see Table 2). As can be seen from Table 4 (Model II), on days without school $F \& V$ deliveries, the thrice weekly intervention group's increase was significantly larger than the control group's increase (by $\gamma_{101}=0.75$ ). That is, the thrice weekly intervention group increased their $\mathrm{F} \& \mathrm{~V}$ intake by $0.78\left(\gamma_{100}+\gamma_{101}\right)$ frequencies/d, from 0.85 frequencies/d at pre-intervention to 1.63 frequencies/d at post-intervention. In a similar vein, the twice weekly intervention group's increase was significantly larger than the control group's intake (by $\gamma_{102}=0.48$ ). That is, the twice weekly intervention group increased their $\mathrm{F} \& \mathrm{~V}$ intake by $0.51\left(\gamma_{100}+\gamma_{102}\right)$ frequencies/d, from 0.91 frequencies/d at pre-intervention to 1.42 frequencies/d at post-intervention. The proportional reduction in level-3 variance of slopes for period $\left(u_{10 j}\right)$ was $45 \cdot 47 \%$. Adjusting for level-2 (lunch, gender, SES) and level-3 (grade) covariates as described above confirmed the stability of the results and changed the corresponding coefficients $\left(\gamma_{101}\right.$ and $\left.\gamma_{102}\right)$ only slightly (Table 4 , Modell III).

Regarding the effect on days without school F\&V deliveries, additional exploratory analyses revealed no subgroups that benefited more or less from participation in

Table 4 Intervention effect on days without fruit and vegetable (F\&V) deliveries of the European School Fruit Scheme in North RhineWestphalia, Germany, on the F\&V intake of third and fourth grade children $(n 664)$ from twelve primary schools, beginning to the end of the $2012 / 13$ school year

\begin{tabular}{|c|c|c|c|c|c|c|c|c|c|}
\hline & \multicolumn{6}{|c|}{ Fixed part } & \multicolumn{3}{|c|}{ Random part } \\
\hline & Coef. & Est. & SE & $t$ & $P$ value & df & Coef. & Var. & $P$ value \\
\hline \multicolumn{10}{|l|}{ Model I } \\
\hline Intercept & $\gamma_{000}$ & 1.00 & 0.06 & $16 \cdot 82$ & $<0.001$ & 47 & $u_{00 j}$ & 0.13 & $<0.001$ \\
\hline \multicolumn{10}{|l|}{ For period slope } \\
\hline Intercept & $\gamma_{100}$ & 0.41 & 0.08 & $5 \cdot 22$ & $<0.001$ & 47 & $u_{10 j}$ & 0.21 & $<0.001$ \\
\hline \multicolumn{10}{|l|}{ Model II } \\
\hline Intercept & $\gamma_{000}$ & 1.25 & 0.09 & 13.61 & $<0.001$ & 45 & $u_{00 j}$ & 0.09 & $<0.001$ \\
\hline Thrice weekly intervention & $\gamma_{001}$ & -0.40 & 0.13 & -3.03 & 0.004 & 45 & & & \\
\hline \multirow{2}{*}{\multicolumn{10}{|c|}{ For period slope }} \\
\hline & & & & & & & & & \\
\hline Intercept & $\gamma_{100}$ & 0.03 & 0.11 & 0.29 & 0.777 & 45 & $u_{10 j}$ & 0.11 & $<0.001$ \\
\hline Thrice weekly intervention & $\gamma_{101}$ & 0.75 & 0.17 & 4.53 & $<0.001$ & 45 & & & \\
\hline Twice weekly intervention & $\gamma_{102}$ & 0.48 & 0.15 & $3 \cdot 16$ & 0.003 & 45 & & & \\
\hline \multicolumn{10}{|l|}{ Model III } \\
\hline Intercept & $\gamma_{000}$ & $1 \cdot 20$ & 0.08 & 14.78 & $<0.001$ & 44 & $u_{00 j}$ & 0.08 & $<0.001$ \\
\hline Thrice weekly intervention & $\gamma_{001}$ & -0.29 & 0.12 & -2.42 & 0.02 & 44 & & & \\
\hline Twice weekly intervention & $\gamma_{002}$ & -0.31 & 0.11 & -2.79 & 0.008 & 44 & & & \\
\hline Grade & $\gamma_{003}$ & -0.36 & 0.09 & -3.84 & $<0.001$ & 44 & & & \\
\hline Lunch at home & $\gamma_{010}$ & -0.31 & 0.06 & $-5 \cdot 25$ & $<0.001$ & 513 & & & \\
\hline Gender & $\gamma_{020}$ & 0.32 & 0.06 & 5.59 & $<0.001$ & 513 & & & \\
\hline Low SES & $\gamma_{030}$ & $-0 \cdot 18$ & 0.08 & $-2 \cdot 25$ & 0.025 & 513 & & & \\
\hline Middle SES & $\gamma_{040}$ & $-0 \cdot 19$ & 0.07 & $-2 \cdot 81$ & 0.005 & 513 & & & \\
\hline \multicolumn{10}{|l|}{ For period slope } \\
\hline Intercept & $\gamma_{100}$ & 0.03 & 0.10 & 0.32 & 0.754 & 44 & $u_{10 j}$ & 0.10 & $<0.001$ \\
\hline Thrice weekly intervention & $\gamma_{101}$ & 0.76 & 0.16 & $4 \cdot 70$ & $<0.001$ & 44 & & & \\
\hline Twice weekly intervention & $\gamma_{102}$ & 0.50 & 0.14 & 3.60 & $<0.001$ & 44 & & & \\
\hline Grade & $\gamma_{103}$ & 0.23 & 0.12 & 1.90 & 0.064 & 44 & & & \\
\hline Lunch at home & $\gamma_{110}$ & 0.14 & 0.09 & 1.57 & 0.117 & 513 & & & \\
\hline Gender & $\gamma_{120}$ & 0.18 & 0.09 & 1.90 & 0.063 & 47 & $u_{12 j}$ & 0.10 & 0.046 \\
\hline Low SES & $\gamma_{130}$ & -0.09 & 0.12 & -0.71 & 0.478 & 513 & & & \\
\hline Middle SES & $\gamma_{140}$ & 0.07 & 0.10 & 0.70 & 0.482 & 513 & & & \\
\hline
\end{tabular}

Coef., coefficient; Est., estimate; Var., variance components; SES, socio-economic status.

The variance of the level-1 residual $\left(e_{t i j}\right)$ was estimated to be 0.89 in Model I and Model II and 0.90 in Model III. The variance of the level-2 residual of intercepts $\left(r_{0 i j}\right)$ was 0.26 in Models I and II and 0.20 in Model III. The variance of the level-2 residual of slopes for period $\left(r_{1 i j}\right)$ was 0.34 in Models I and II and 0.30 in Model III. 
the programme. That is, no significant cross-level interactions were found.

\section{Comparison of the effectiveness of different distribution frequencies of school fruit and vegetable deliveries}

As presented in Tables 2 to 4 , school F\&V deliveries at a frequency of thrice as well as twice weekly significantly increased daily F\&V intake of children participating in the SFVS compared with the control group on days with and without school F\&V deliveries. Additionally, we aimed to test whether the two intervention groups (twice $v$. thrice weekly deliveries) differed with respect to the increase in F\&V intake from pre- to post-intervention. The results showed that the two intervention groups did not differ with respect to the F\&V increase over time (overall effect: $P=0.207$; effect on days with school F\&V deliveries: $P=0.274$; effect on days without school $\mathrm{F} \& \mathrm{~V}$ deliveries: $P=0.151$; see online supplementary material, Supplemental Tables 3-5).

\section{Discussion}

The data demonstrate that the current implementation of the SFVS NRW with thrice weekly F\&V deliveries as well as an adjustment to a twice weekly delivery is successful in increasing children's daily F\&V intake. Thus, our results underline the effectiveness of increased availability and repeated exposure in mediating the dietary behaviour of participating children, as theory and previous empirical research have suggested ${ }^{(25,48-50)}$.

A systematic review of published and unpublished studies identified fifteen studies focusing on interventions designed to increase F\&V intake of 5- to 18-year-old children and adolescents. Of those none revealed a detrimental effect on F\&V intake while ten of the studies found a significant effect on children's F\&V intake ranging from 0.3 to 0.99 servings $/ \mathrm{d}^{(51)}$. Thus, our estimates of 0.96 frequencies/d for the thrice weekly intervention group and 0.75 frequencies/d for the twice weekly intervention group are in line with previous research. Considering that intervention approaches vary greatly between studies and that we used $F \& V$ frequencies rather than servings to quantify the effect on F\&V intake, our findings are most comparable to the results of Methner et al. ${ }^{(38)}$. Those authors carried out the first evaluation study of the SFVS NRW and found an overall effect of 0.77 frequencies/d. Obviously, our results show a slightly greater effect compared with the estimates of Methner et al. ${ }^{(38)}$, even though the authors investigated the effectiveness of the SFVS NRW with daily F\&V deliveries. As our study used three repeated $24 \mathrm{~h}$ dietary recalls instead of only one as in Methner et al.'s study ${ }^{(38)}$, a possible explanation for the small differences could be that the potential for recall bias was lower in our study. Additionally, the study by Methner et $a l .{ }^{(38)}$ was carried out in the first year of SFVS NRW. Guidelines and support for schools in implementing nutrition education programmes have improved considerably in the subsequent years and likely increased the application of those measures in the participating schools. This argument is supported by other studies showing that multicomponent programmes are more effective in changing dietary behaviour of children than single measures ${ }^{(51,52)}$.

Interestingly, both types of interventions (twice and thrice weekly $F \& V$ deliveries) investigated in our study showed a similar increase in F\&V intake from pre- to postintervention and evidence for differential effectiveness was low. In contrast, an earlier evaluation study of the SFVS arrived at the conclusion that a distribution frequency of one to two times weekly is insufficient ${ }^{(53)}$. Against this background, our results should be interpreted with caution in order to avoid premature conclusions. Besides subgroup analysis of the covariates included in our study, further analysis should also consider potential moderators of F\&V intake such as personal and social determinants. The latter have been shown to influence F\&V intake of children ${ }^{(25,54,55)}$ and therefore might also moderate the effect of the SFVS NRW, even though determinants of $F \& V$ intake have not often been studied in the context of moderation effects ${ }^{(56)}$. Differentiating the results according to days with and without school F\&V deliveries revealed that on days with F\&V deliveries children in the twice weekly intervention group who usually eat lunch at school tended to benefit more from the F\&V deliveries compared with children who usually eat lunch at home. This result clearly shows a first indication for differential effectiveness and is most probably attributable to the fact that, as stated by school principals, leftovers of $F \& V$ in the twice weekly intervention group are distributed to children in the school canteen at lunch time. This interpretation is also supported by our finding that on days without school F\&V deliveries eating lunch at school had no effect on children's increase in F\&V intake. As those children eating lunch at school showed a higher F\&V intake at pre-intervention, this result may not be desired by the initiators of the SFVS NRW, because it advantages children with a higher initial F\&V intake. Nevertheless, the appropriateness of this practice depends strongly on the nutritional value of food provided in the specific school canteen, which at present varies considerably across different schools in Germany ${ }^{(57)}$. Besides these findings, additional exploratory analyses provided little evidence for the existence of differential effects of the SFVS NRW with respect to class and child characteristics. This holds particularly true for grade and gender. Also SES showed no significant interaction effect in the overall model, a result which is in line with previous research analysing the effects of the SFVS NRW ${ }^{(38)}$. Exclusively investigating the effect of the SFVS NRW on days with F\&V deliveries revealed that children with a middle SES in the twice 
weekly intervention group benefit less from the scheme compared with those with a high SES. A similar effect was not detected for children with a middle SES, thus an interpretation of this result proves to be difficult. Regarding the thrice weekly intervention group not even low evidence for differential effectiveness was found, meaning that there were no subgroups benefiting more from the school F\&V deliveries. This can be positively interpreted, but it also implies that it was not possible to give special support to subgroups, such as boys or children from homes with low SES who are known to eat less F\&V $\mathrm{V}^{(58,59)}$ and had significantly lower pre-intervention scores in our study as well. However, this is not to say that these subgroups did not benefit from the programme.

Overall our results indicate that particularly the thrice weekly F\&V deliveries are an effective instrument for increasing the $\mathrm{F} \& \mathrm{~V}$ intake of all children participating in the SFVS NRW. It therefore seems likely that the SFVS has relative advantages compared with other measures aiming to improve the dietary habits of the population. For example, previous research has indicated that high-risk approaches such as dietary advice typically widen socioeconomic inequalities ${ }^{(60)}$. This also seems to be true in general for interventions that require individuals to use a high level of agency to benefit ${ }^{(61)}$. In contrast, those that require fewer agencies are considered to be more effective because they reach broader segments of society ${ }^{(62)}$.

Our results also indicate that the SFVS NRW was successful in increasing $F \& V$ intake of children on days without F\&V deliveries. Regarding the thrice weekly intervention group, this result was not surprising, as school principals stated in interviews that they distribute the delivered F\&V over the whole school week. This also implies that the effect in the thrice weekly intervention group on days without F\&V deliveries should be interpreted with caution, as it likely not indicates long-term effects, but rather reflects the increase in F\&V availability due to the F\&V leftovers from the previous day. Remarkable, however, is that children of the twice weekly intervention group increased their $\mathrm{F} \& \mathrm{~V}$ intake on days without school F\&V deliveries to a comparable degree. As mentioned before, school principals of all four schools in this group clearly stated that they provide the F\&V only on two fixed days during one school week. Hence, these findings provide first evidence that the SFVS NRW can have a sustainable effect on children's dietary behaviour apart from the direct effect of F\&V deliveries. Nevertheless, it should be noted that $F \& V$ intake of children increased considerably more on days with school F\&V deliveries, supporting the importance of accessibility and availability as predictors for $\mathrm{F} \& \mathrm{~V}$ intake $\mathrm{in}^{(23-25)}$.

In conclusion, even though there might be some determinants which have not yet been taken into account in terms of differential effectiveness, from the present state of analysis, the SFVS NRW reaches all considered subgroups, a result which holds particularly true for the thrice weekly implementation form and represents an advantage over many other strategies aiming to improve dietary behaviour. However, it should be noted that a clear limitation of our study is the non-randomization of schools to the study groups. Although differences between the groups were controlled by including respective variables as covariates and by applying a statistical method which best fits the data structure, there might be some other factors not addressed in our analysis. For example, the intensity and quality of accompanying measures could have had an influence on the effectiveness of both implementation forms and might be different between the study groups. However, regarding the selection criterion 'nutrition education measures', all schools are obliged to implement respective measures once entering the programme. Thus, this holds for schools originally selected by the ministry as well as those that were later included due to our study. We are therefore confident that this criterion will not influence our findings. Additionally, a pre-post design where subjects are not randomly assigned to groups is most common in evaluation research ${ }^{(63,64)}$. In terms of limitations, it should be also mentioned that measuring dietary intake is always a challenge and requires validated and reliable instruments. We addressed this issue by selecting an existing and validated $24 \mathrm{~h}$ dietary recall proven to be reliable for the specific age group of our sample and used before in the context of evaluating the effect of the SFS NRW ${ }^{(38,41)}$. We also tried to reduce the potential for recall bias by using three instead of only one $24 \mathrm{~h}$ dietary recall at pre- and postintervention. However, two $24 \mathrm{~h}$ dietary recalls at each time point were filled in by the children in the absence of the research team. Although teachers were given specific instructions on how to guide children while completing the questionnaire, we can neither completely exclude that teachers influenced children's answers nor that some children had problems to remember the foods and drinks eaten on the previous day. However, as instruments and respondents did not change from pre- to post-intervention, we expect high comparability of pre- and post-intervention measurements.

\section{Conclusions}

Our results clearly indicate that the current implementation of the SFVS NRW of providing children thrice weekly with school F\&V deliveries is successful in increasing children's F\&V intake. The findings also suggest that the impact of the SFVS NRW in its present form does not discriminate subgroups of children. This, however, also implies that those groups known to have a low intake level of $\mathrm{F} \& \mathrm{~V}$, such as boys or children from homes with a low SES, are not specifically supported by the programme. Reducing the distribution frequency of $F \& V$ to twice weekly deliveries is, according to the first findings presented here, similarly effective in positively influencing children's F\&V intake, but there is also some evidence that this implementation form would advantage children eating 
lunch in the school canteen. The findings also show that children's F\&V intake increases significantly on days without F\&V deliveries. This result provides a first indication of the positive long-term effects of the scheme.

Assuming a given budget, the twice weekly implementation would allow to provide more children with $\mathrm{F} \& \mathrm{~V}$ in school. However, a recommendation for one or the other implementation needs to consider the potential discriminating effect as well as the longer-term impact of both intervention types and thus should be based on further research.

\section{Acknowledgements}

Acknowledgements: The authors wish to thank the children and teachers in the schools in NRW that served as study sites for the analysis. They are grateful to Dr Sarah Methner for her thoughtful comments and ideas in the collection and coding of the data. They also want to express appreciation to Gesa Maschkowski who adapted the $24 \mathrm{~h}$ dietary recall used in this study to the German school system. Financial support: This work was funded by the North RhineWestphalian Ministry for Climate Protection, Environment, Agriculture, Conservation and Consumer Protection (MKULNV). MKULNV had no role in the design, analysis or writing of this article. Conflict of interest: All authors state that there are no conflicts of interest. Authorship: M.H. and J.H. developed the research question and designed the study, J.H. conducted the data collection with organizational support from M.H. The coding form was developed by J.H. based on previous work in the department in Bonn. J.H. supervised and controlled the coding and conducted the statistical analyses jointly with T.L. J.H. wrote the first draft of the manuscript. The methodology and results parts were extended and revised by T.L. M.H. revised the manuscript with a focus on the introduction, discussion and conclusion parts. All authors made substantive intellectual contributions to the scientific content and approved the final manuscript. Ethics of buman subject participation: Written informed consent was obtained from all parents of included children. Anonymized number codes allowed to link baseline data to follow-up data for each individual. The study and data handling were approved by the Ministry of Education and the MKULNV of NRW as well as by the data protection officer of the University of Bonn.

\section{Supplementary material}

To view supplementary material for this article, please visit https://doi.org/10.1017/S1368980017003949

\section{References}

1. Lobstein T, Baur L \& Uauy R (2004) Obesity in children and young people: a crisis in public health. Obes Rev 5, Suppl. 1, 4-104.
2. Ng M, Fleming T, Robinson M et al. (2014) Global, regional, and national prevalence of overweight and obesity in children and adults during 1980-2013: a systematic analysis for the Global Burden of Disease Study 2013. Lancet $\mathbf{3 8 4}$, 766-781.

3. Brettschneider A-K, Schaffrath Rosario A, Kuhnert R et al. (2015) Updated prevalence rates of overweight and obesity in 11- to 17-year-old adolescents in Germany. Results from the telephone-based KiGGS Wave 1 after correction for bias in self-reports. BMC Public Health 15, 1101.

4. Abarca-Gómez L, Abdeen ZA, Hamid ZA et al. (2017) Worldwide trends in body-mass index, underweight, overweight, and obesity from 1975 to 2016: a pooled analysis of 2416 population-based measurement studies in 128.9 million children, adolescents, and adults. Lancet $\mathbf{3 9 0}$, 2627-2642.

5. Fletcher S, Wright C, Jones A et al. (2017) Tracking of toddler fruit and vegetable preferences to intake and adiposity later in childhood. Matern Child Nutr 13, e12290.

6. Lien N, Lytle LA \& Klepp KI (2001) Stability in consumption of fruit, vegetables, and sugary foods in a cohort from age 14 to age 21. Prev Med 33, 217-226.

7. Baranowski T, Mendlein J, Resnicow K et al. (2000) Physical activity and nutrition in children and youth: an overview of obesity prevention. Prev Med 31, Suppl. 2, S1-S10.

8. Pařízková J (2009) Nutrition, Physical Activity, and Health in Early Life, 2nd ed. Boca Raton, FL: Taylor \& Francis.

9. Chan RSM \& Woo J (2010) Prevention of overweight and obesity: how effective is the current public health approach. Int J Environ Res Public Health 7, 765-783.

10. Afshin A, Forouzanfar MH, Reitsma MB et al. (2017) Health effects of overweight and obesity in 195 countries over 25 years. $N$ Engl J Med 377, 13-27.

11. Müller-Riemenschneider F, Reinhold T, Berghöfer A et al. (2008) Health-economic burden of obesity in Europe. Eur J Epidemiol 23, 499-509.

12. Lytle LA, Jacobs DR, JR, Perry CL et al. (2002) Achieving physiological change in school-based intervention trials: what makes a preventive intervention successful? BrJ Nutr 88, 219-221.

13. Lobstein T \& Baur LA (2005) Policies to prevent childhood obesity in the European Union. Eur J Public Health 15, 576-579.

14. Hassan A (editor) (2015) School Nutrition and Activity: Impacts on Well-Being. Oakville, ON: Apple Academic Press.

15. Perez-Cueto FJA, Aschemann-Witzel J, Shankar B et al. (2012) Assessment of evaluations made to healthy eating policies in Europe: a review within the EATWELL Project. Public Health Nutr 15, 1489-1496.

16. de Bourdeaudhuij I, van Cauwenberghe E, Spittaels $\mathrm{H}$ et al. (2011) School-based interventions promoting both physical activity and healthy eating in Europe: a systematic review within the HOPE project. Obes Rev 12, 205-216.

17. Rolls BJ, Ello-Martin JA \& Tohill BC (2004) What can intervention studies tell us about the relationship between fruit and vegetable consumption and weight management? Nutr Rev 62, 1-17.

18. Ledoux TA, Hingle MD \& Baranowski T (2011) Relationship of fruit and vegetable intake with adiposity: a systematic review. Obes Rev 12, e143-e150.

19. He K, Hu FB, Colditz GA et al. (2004) Changes in intake of fruits and vegetables in relation to risk of obesity and weight gain among middle-aged women. Int J Obes Relat Metab Disord 28, 1569-1574.

20. Vioque J, Weinbrenner T, Castello A et al. (2008) Intake of fruits and vegetables in relation to 10 -year weight gain among Spanish adults. Obesity (Silver Spring) 16, 664-670.

21. Yngve A, Wolf A, Poortvliet E et al. (2005) Fruit and vegetable intake in a sample of 11-year-old children in 
9 European countries: The Pro Children Cross-sectional Survey. Ann Nutr Metab 49, 236-245.

22. Borrmann A \& Mensink GBM (2015) Obst- und Gemusekonsum von Kindern und Jugendlichen in Deutschland: Ergebnisse der KiGGS-Welle 1. Bundesgesundheitsblatt Gesundheitsforschung Gesundheitsschutz 58, 1005-1014.

23. Evans CEL, Christian MS, Cleghorn CL et al. (2012) Systematic review and meta-analysis of school-based interventions to improve daily fruit and vegetable intake in children aged 5 to 12 y. Am J Clin Nutr 96, 889-901.

24. de Sa J \& Lock K (2008) Will European agricultural policy for school fruit and vegetables improve public health? A review of school fruit and vegetable programmes. Eur J Public Health 18, 558-568.

25. Rasmussen M, Krolner R, Klepp K-I et al. (2006) Determinants of fruit and vegetable consumption among children and adolescents: a review of the literature. Part I: quantitative studies. Int J Behav Nutr Phys Act 3, 22.

26. Wolnicka K, Taraszewska AM, Jaczewska-Schuetz J et al. (2015) Factors within the family environment such as parents' dietary habits and fruit and vegetable availability have the greatest influence on fruit and vegetable consumption by Polish children. Public Health Nutr 18, 2705-2711.

27. Wyse R, Wolfenden L \& Bisquera A (2015) Characteristics of the home food environment that mediate immediate and sustained increases in child fruit and vegetable consumption: mediation analysis from the Healthy Habits cluster randomised controlled trial. Int J Behav Nutr Phys Act 12, 118.

28. Martin CR, Watson RR \& Preedy VR (editors) (2011) Handbook of Behavior, Food and Nutrition. New York: Springer.

29. Birch LL, Gunder L, Grimm-Thomas K et al. (1998) Infants' consumption of a new food enhances acceptance of similar foods. Appetite 30, 283-295.

30. Gerrish CJ \& Mennella JA (2001) Flavor variety enhances food acceptance in formula-fed infants. Am J Clin Nutr $\mathbf{7 3}$, 1080-1085.

31. Loewen R \& Pliner P (1999) Effects of prior exposure to palatable and unpalatable novel foods on children's willingness to taste other novel foods. Appetite 32, 351-366.

32. Pliner P \& Stallberg-White C (2000) 'Pass the ketchup, please': familiar flavors increase children's willingness to taste novel foods. Appetite 34, 95-103.

33. Shepherd J, Harden A, Rees R et al. (2006) Young people and healthy eating: a systematic review of research on barriers and facilitators. Health Educ Res 21, 239-257.

34. European Commission (2015) School Fruit Scheme: EU initiative. http://www.ec.europa.eu/agriculture/sfs/europeancommission/index_en.htm (accessed February 2015).

35. European Commission (2012) EU School Fruit Scheme North Rhine-Westphalia: North Rhine-Westphalia - Regional Strategy for the EU School Fruit Scheme. http://www. ec.europa.eu/agriculture/sfs/documents/de_national_strategy_-nordhein-westfallen_-_2012-2013_en.pdf (accessed February 2015).

36. Ministerium für Klimaschutz, Umwelt, Landwirtschaft, Natur- und Verbraucherschutz NRW (2015) EU-Schulobstund -gemüseprogramm NRW: Daten und Fakten. http:// www.schulobst.nrw.de/allgemeine-informationen/daten-undfakten.html (accessed February 2015).

37. Ministerium für Klimaschutz, Umwelt, Landwirtschaft, Naturund Verbraucherschutz NRW (2011) Regionale Strategie Nordrhein-Westfalens für das EU-Schulobstprogramm NRW Durchführungszeitraum: 1. https://ec.europa.eu/agriculture/ sites/agriculture/files/sfs/documents/de_national_strategy_-nordhein-westfallen_-_2012-2013_de.pdf (accessed October 2017).

38. Methner S, Maschkowski G \& Hartmann M (2017) The European School Fruit Scheme: impact on children's fruit and vegetable consumption in North Rhine-Westphalia, Germany. Public Health Nutr 20, 542-548.
39. Fernández-Alvira JM, Börnhorst C, Bammann K et al. (2015) Prospective associations between socio-economic status and dietary patterns in European children: the Identification and Prevention of Dietary- and Lifestyle-induced Health Effects in Children and Infants (IDEFICS) Study. Br J Nutr $\mathbf{1 1 3}, 517-525$.

40. European Commission, Directorate-General for Agriculture and Rural Development (2015) Report on the Results of the Evaluation of the School Fruit and Vegetables and School Milk Scheme against the Principles of Subsidiarity, Proportionality and Better Regulation. http://www.ec.europa. eu/agriculture/sites/agriculture/files/school-scheme/assessment/ final-report_en.pdf (accessed January 2017).

41. Edmunds LD \& Ziebland S (2002) Development and validation of the Day in the Life Questionnaire (DILQ) as a measure of fruit and vegetable questionnaire for 7-9 year olds. Health Educ Res 17, 211-220.

42. Böhm A, Ellsässer G \& Lüdecke K (2007) Der Brandenburger Sozialindex: ein Werkzeug für die Gesundheits- und Sozialberichterstattung auf Landes- und kommunaler Ebene bei der Analyse von Einschülerdaten. Gesundheitswesen 69, 555-559.

43. Raudenbush SW \& Bryk AS (2010) Hierarchical Linear Models: Applications and Data Analysis Methods, 2nd ed. Thousand Oaks, CA: SAGE Publications, Inc.

44. Bell BA, Morgan GB, Schoeneberger JA et al. (2014) How low can you go? Methodology 10, 1-11.

45. Lischetzke T, Reis D \& Arndt C (2015) Data-analytic strategies for examining the effectiveness of daily interventions. J Occup Organ Psychol 88, 587-622.

46. Enders CK \& Tofighi D (2007) Centering predictor variables in cross-sectional multilevel models: a new look at an old issue. Psychol Methods 12, 121-138.

47. Snijders TAB \& Bosker RJ (2012) Multilevel Analysis: An Introduction to Basic and Advanced Multilevel Modeling, 2nd ed. Los Angeles, CA: SAGE Publications, Inc.

48. Gochman DS (editor) (1997) Handbook of Health Behavior Research. New York: Plenum Press.

49. Jago R, Baranowski T \& Baranowski JC (2007) Fruit and vegetable availability: a micro environmental mediating variable? Public Health Nutr 10, 681-689.

50. Reinaerts E, Nooijer J, de, Candel M et al. (2007) Increasing children's fruit and vegetable consumption: distribution or a multicomponent programme? Public Health Nutr 10, 939-947.

51. Knai C, Pomerleau J, Lock K et al. (2006) Getting children to eat more fruit and vegetables: a systematic review. Prev Med 42, 85-95.

52. van Cauwenberghe E, Maes L, Spittaels H et al. (2010) Effectiveness of school-based interventions in Europe to promote healthy nutrition in children and adolescents: systematic review of published and 'grey' literature. $\mathrm{Br} \mathrm{J}$ Nutr 103, 781-797.

53. Elles A, Kliebisch C, Becker A et al. (2012) Evaluation of the European School Fruit Scheme. Bonn and Luxembourg: AFC Management Consulting AG and CO CONCEPT Marketing Consulting; available at http://www.ec.europa.eu/ agriculture/sites/agriculture/files/evaluation/market-andincome-reports/2012/school-fruit-scheme/fulltext_en.pdf

54. Reinaerts E, de Nooijer J, van de Kar A et al. (2006) Development of a school-based intervention to promote fruit and vegetable consumption: exploring perceptions among 4-to-12-year old children and their parents. Health Educ 106, 345-356.

55. Haß J \& Hartmann M (2017) What determines the fruit and vegetables intake of primary school children? - An analysis of personal and social determinants. Appetite 120, 82-91.

56. Yildirim M, van Stralen MM, Chinapaw MJM et al. (2011) For whom and under what circumstances do school-based energy balance behavior interventions work? Systematic review on moderators. Int J Pediatr Obes 6, e46-e57. 
57. Bundesministerium für Ernährung und Landwirtschaft (2016) Verpflegung in Schulen. http://www.in-form.de/ fileadmin/redaktion/Publikationen/pdfs/6_INFORM_Monitor_ Verpflegung_Schule.pdf (accessed November 2016).

58. Fismen A-S, Smith ORF, Torsheim T et al. (2014) A school based study of time trends in food habits and their relation to socio-economic status among Norwegian adolescents, 2001-2009. Int J Behav Nutr Phys Act 11, 115.

59. Leech RM, McNaughton SA \& Timperio A (2014) The clustering of diet, physical activity and sedentary behavior in children and adolescents: a review. Int J Behav Nutr Phys Act 11, 4

60. Capewell S \& Graham H (2010) Will cardiovascular disease prevention widen health inequalities? PLOS Med 7, e1000320.
61. Adams J, Mytton O, White M et al. (2016) Why are some population interventions for diet and obesity more equitable and effective than others? The role of individual agency. PLoS Med 13, e1001990.

62. Frieden TR (2010) A framework for public health action: the health impact pyramid. Am J Public Health 100, 590-595.

63. Weiss CH (1972) Evaluation Research: Methods for Assessing Program Effectiveness. Englewood Cliffs, NJ: Prentice-Hall.

64. Green JL, Camilli G \& Elmore PB (2012) Handbook of Complementary Methods in Education Research. London: Taylor \& Francis. 